Ann. Abeille, I968, 11 (I), 5-I4.

\title{
ÉVOLUTION DE LA CONSOMMATION D'OXYGÈNE AU COURS DU DÉVELOPPEMENT LARVAIRE DES OUVRIÈRES DE BOMBUS TERRESTRIS L. (HYMENOPTERA, APOIDEA, BOMBINA).
}

\author{
A. POUVREAU \\ Station de Recherches sur l'Abeille et les Insectes sociaux, 91 -Bures-sur-Yvette \\ Institut national de la Recherche agronomique
}

SOMMAIRE

'L'auteur étudie les échanges respiratoires chez les larves de Bombus terrestris. Les mesures respirométriquës; effectuées à l'aide de l'appareil de Warburg, indiquent la consommation d'oxygène des larves en fonction du stade et du poids moyen. Les résultats du niveau de l'intensité respiratoire sont comparés à ceux enregistrés chez les larves d'Apis mellifica.

\section{INTRODUCTION}

Si l'étude de la consommation d'oxygène chez les Hyménoptères sociaux a retenu l'attention d'un certain nombre d'auteurs, les données concernant les échanges respiratoires des stades immatures chez ces insectes se limitent aux travaux effectués sur Apis mellifica L.

MELAMPY et WILLIs (I939), travaillant sur des larves et des nymphes d'Apis mellifica, ont mesuré, à l'aide de la technique de Barcroft-Warburg, la consommation d'oxygène et la production de gaz carbonique, ainsi que le quotient respiratoire, de reines et d'ouvrières.

Quelques travaux font mention de mesures de la consommation d'oxygène chez des larves de mâles et d'ouvrières d'Apis mellifica : AlLEN (I959); Inoue. (I964); Hukusima et Inoue (I964). Mais, ces deux derniers auteurs ont comparé dẻ plus l'intensité des échanges respiratoires chez deux espèces : Apis mellifica $\mathrm{L}_{1}$. et Apis cerana $F$., au cours du développement larvaire et nymphal, puis chez les adultes.

Les relations entre l'intensité respiratoire et les conditions alimentaires chez les larves d'Apis mellifica ont fait 1'objet d'études récentes. C'est ainsi que SHuEr et Dixon (I959) ont pu établir une corrélation entre la quantité de gaz carbonique rejetée par les larves et la composition de différents régimes alimentaires sotımis à celles-ci. En mesurant la consommation d'oxygène de larves âgées de trois jours, élevées sur 
gelée royale, RE,MBor,D et HAvser (I964) ont observé que les larves qui se développaient ultérieurement en reines avaient une consommation d'oxygène beaucoup plus élevée que celles qui étaient destinées à devenir des ouvrières.

Le problème concernant les échanges respiratoires chez les Bourdons n'a jamais été abordé. J'ai donc entrepris une série de mesures de la consommation d'oxygène chez les larves de Bombus au cours des différents stades, afin de comparer le niveau et les variations des échanges respiratoires avec ceux déjà observés chez Apis mellifica, en tenant compte qu'il existe une organisation sociale différente dans les deux genres.

\section{CONDITIONS EXPÉRIMENTALES}

\section{Matériel biologique}

La croissance larvaire a été suivie dans deux colonies de Bombusterrestris, en conditions d'élevage spontanées.

La croissance de Bombus terrestris comporte cinq stades bien déterminés, permettant la connaissance certaine de chaque intermue. Après leur éclosion, les larves, au nombre de 6 à I 6 par cellule, demeurent groupées; puis à partir du cinquième ou sixième jour, elles tissent un mince cocon soyeux, toujours it l'intéricur de la cellule larvaire. Les larves poursuivent leur croissance jusqu'à la mue nymphale, les cocons formant une masse compacte. Ia durée de la vie larvaire est de onze jours environ.

I la période où les expériences ont été réalisées, chaque colonie comprenait 25 à zo ouvrières. Les rempérat ures à l'intérieur des nids oscillaient entre 22 et 2800 ?

\section{Methode d'étude}

Les mesures respirométriques sont effectuées à l'aide de l'appareil de Warburg et de manomètres différeintiels de Summerson à volume co istant. Le gaz carboni jue dégagé par les insectes est absorbé par une solution de potasse (o,6 millilitre) ì ro p. Ioo, dispo iée dans le réservoir latéral de chaque fiole. I.es fioles ont une capacité voisine de $55 \mathrm{ml}$. La température du bain thermostatique est main. tenue constante à $25^{\circ} \mathrm{C}$. Les échanges gazeux sont mesurés pendant une période de trois heures, à raison d'une lecture toutes les heures. Jans les calculs de la consommation d'oxygère, j'ai utilisé la constante du récipient pour le gaz étudié. d'après l'équation de l'yrReit, Burress et STAUFFER (1964):

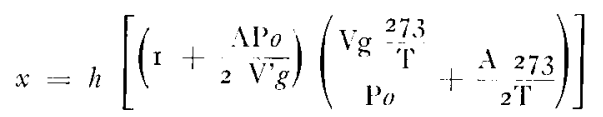

Dans cette constante, on tient compte du volume du récipient, du volune de l'animal, de la température absolue.

A l'intérieur de charque fiole, les larves demeurent dans leur cellule cireuse --- ouverte -- ou bien reposent sur un fragment de leur cellule, afin d'être maintenues dans des conditions aussi naturclles que possible.

Les expériences sont effectuées à la pénombre et toujours à la même heure, de façon à éliminer les oscillations diurnes possibles dans la consommation d'oxygène. Des essais préliminaires sur les échanges respiratoires des larves — à l'obscurité ou en éclairement naturel - n'ont montré aucune modification sensible de l'intensité respiratoire.

Les larves sont pesées à la fin de chaque expérience de façon à réduire au minimum le maniement des insectes avant les mesures respirométriques.

A l'exception des larves du dernier stade qui ont une taille suffisante pour être disposées indivi. duellement dans chaque fiole, il est nécessaire - pour les larves de quatre premiers stades — de constituer des lots de cinc individus, afin de permettre des mesures de consommation d'oxygène avec une précision satisfaisante. J'ai utilisé pour chaque lot des animaux extraits de la même cellule larvaire, de poids très voisins. Ĺes prélèvements de larves sont effectués dans des couvées différentes.

Il était important, afin de pouvoir dégager les variations de la consommation d'oxygène pendant la croissance, de ne prélever que des larves se trouvant à un même stade d'intermue, suffisamment éloigné des remaniements caractéristiques de la mue.

Je me suis donc efforcé de prélever des larves à une période où celles-ci étaient supposées avoir compensé par un nouvel apport de nourriture l'appauvrissement dû aux dépenses de la mue précédente, et où les animaux n'avaient pas encore commencé le stockage des réserves devant permettre at mue suivante. 
TABIEAU I

Consomnations d'oxygène de larves ouvrières de Bombus terrestris en fonction du stade laraire et du poids moyen des laries

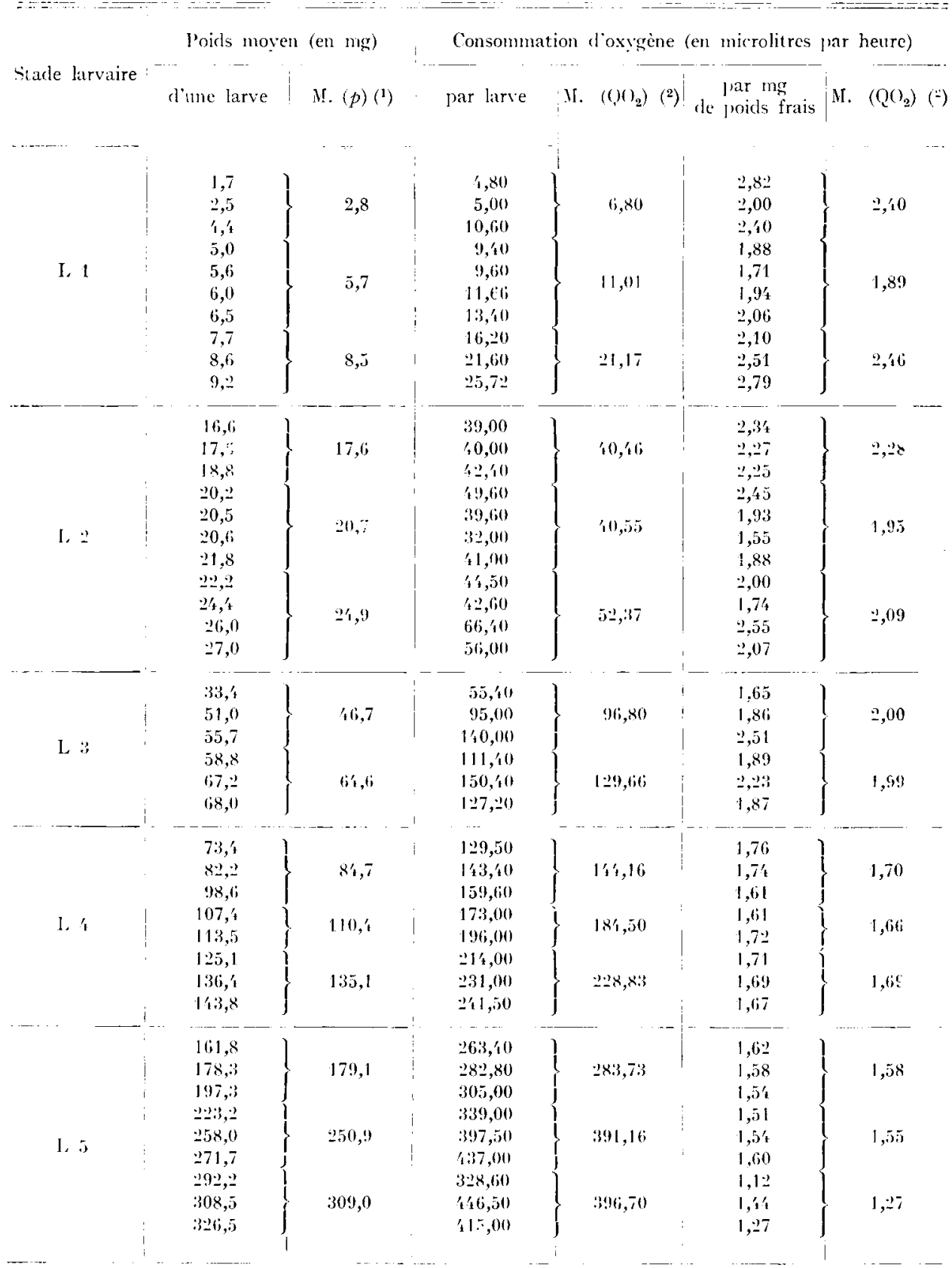

(1) II. (p) : Poids moyen d'un groupe de larves de même âge mais provenant de couvées différentes.

(2) M. $\left(\mathrm{C}_{2}\right)$ : Consommation d'oxygène d'un groupe de larves de mêne âge mais provenant de couvćes clifférentes. 


\section{RÉSULTATS}

Les différentes valeurs de la consommation d'oxygène des larves d'ouvrières de Bombus terrestris sont rapportées dans le tableau suivant, et exprinées en fonction du stade larvaire et clu poids moyen des larves. J'ai calculé les moyennes de poids et de consommation d'oxygène pour des groupes de larves, a fin de montrer l'évolution de la consommation d'oxygène à l'intérieur de chaque stade.

Sur la figure I sont représentées les valeurs de la consommation d'oxygène par larve en fonction du poids moyen de groupes de larves à l'intérieur de chaque stade (exprimées en microlitres d'oxygène par larve et par heure). On constate qu'il y a augmentation de la consommation d'oxygène en rapport avec la croissance pondérale.

Sur la figure 2 , on a porté en abscisse le poids des larves des différents stades, et en ordonnée la consommation d'oxygène. L'allure générale de l'ensemble des points suggère une régression curvilinéaire plutôt que linéaire. İ̀ effet l'ajustement par la méthode des moindres carrés à une courbe parabolicule permet de tester l'influence du terme du second degré quand on a déjà tenu compte de la régression linéaire. La corrélation est très hautement significative, comme le montre de façon équivalente le test $\mathrm{F}$ ou le coefficient de corrélation, autrement dit, il existe une très forte corrélation entre la consommation d'oxygène et la croissance pondérale des larves, dans les limites étudiées.

Analy'se de la iariance

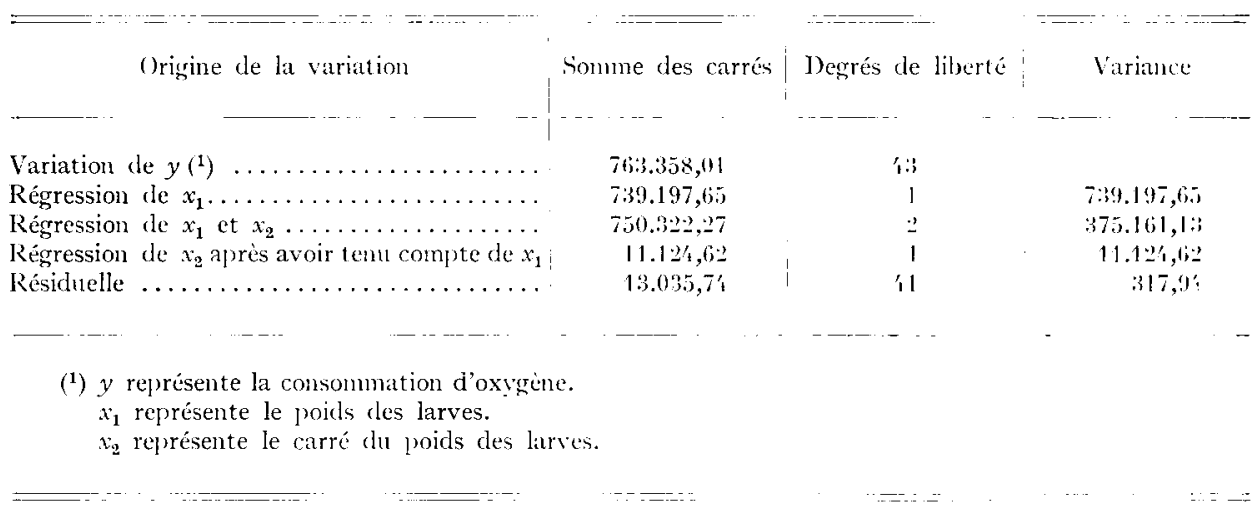

Le rapport des variances est hautement significatif $(F=$ I $\mathrm{I} 79,95)$ pour la régression de $x_{1}$ et $x_{2}$, et significatif $(\mathrm{F}=34,98)$ au seuil I p. IoO pour la régression de $x_{2}$ après avoir tenu compte de $x_{1}$.

La figure 3 montre le niveau de la consommation d'oxygène des larves d'ouvrières des différents stades, rapportée au poids frais des individus (exprimée en microlitres d'oxygène par milligramme et par heure). La consommation d'oxygène par milli- 
Consommotion d'oxygène par larve et par heure

$+1 . \mathrm{O}_{2}$

400
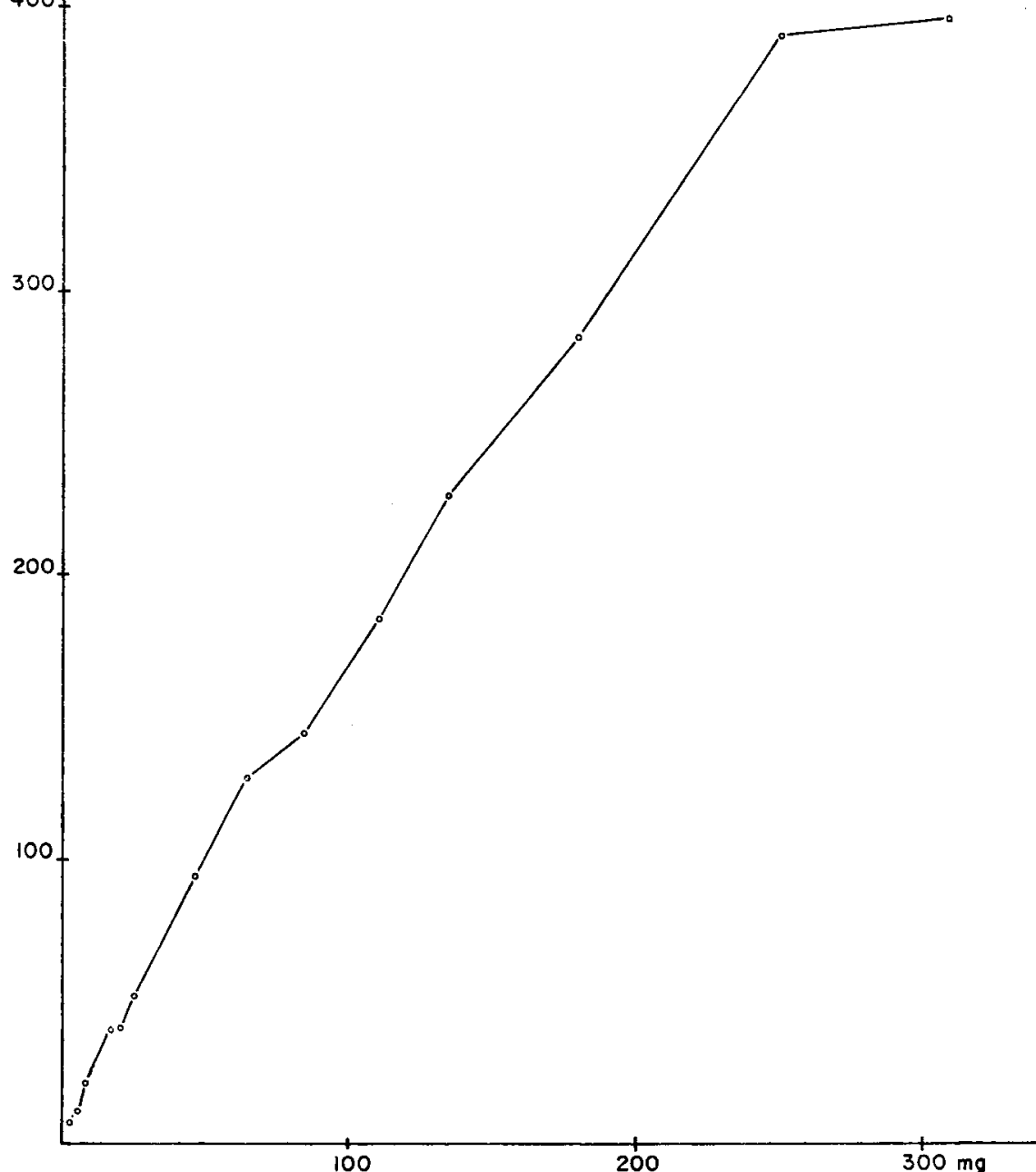

Poids moyens de groupes de larves à l'intérieur de chaque stade

Figure I 
Consommation doxyoenc par larve t por heure

v1. $\mathrm{O}_{2}$

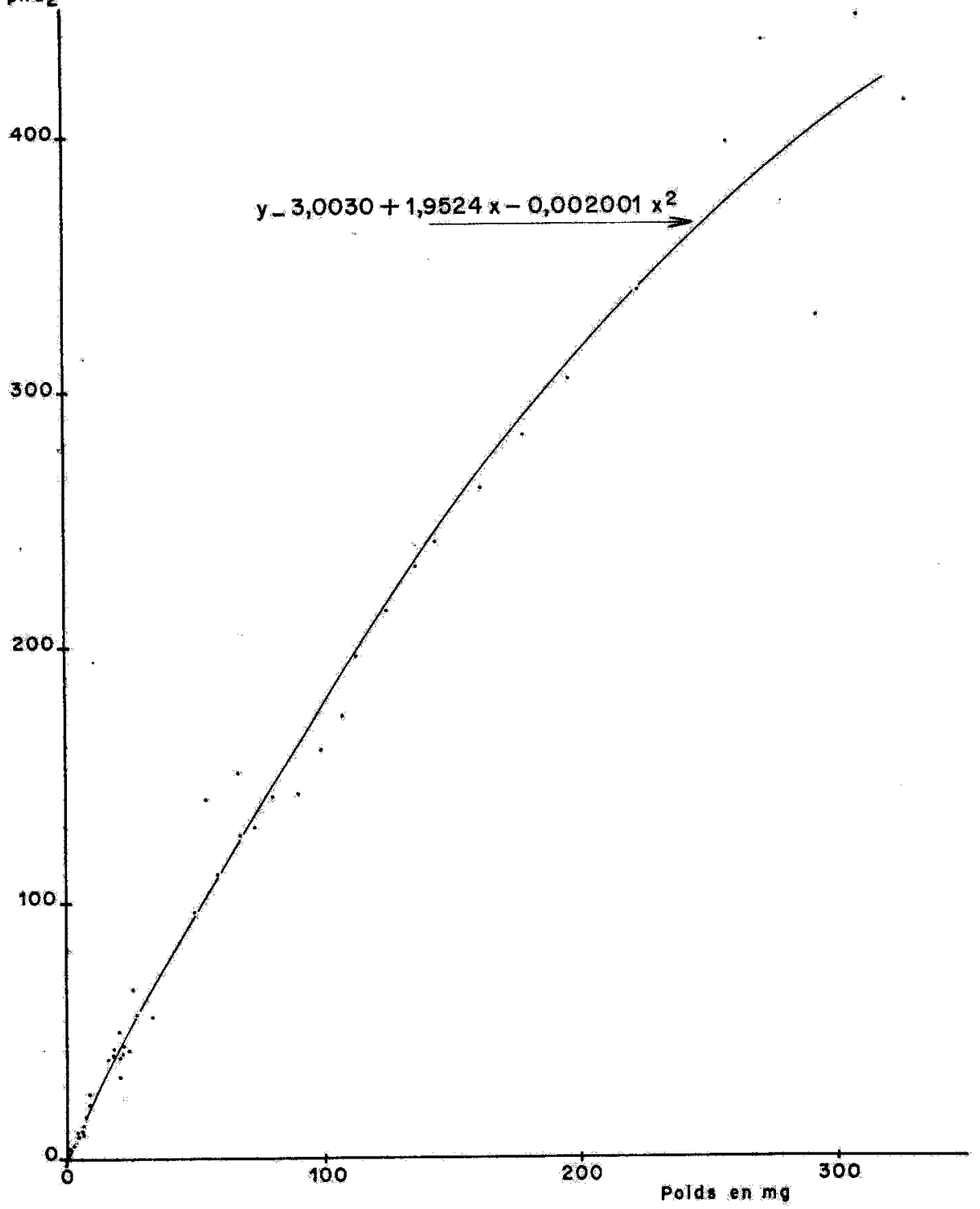

Figure : 
gramme de poids du corps diminue progressivement au fur et à mesure de l'accroissement pondéral des larves, ce qui revient à dire que les plus grosses larves consomment moins d'oxygène par unité de poids que les plus petites.

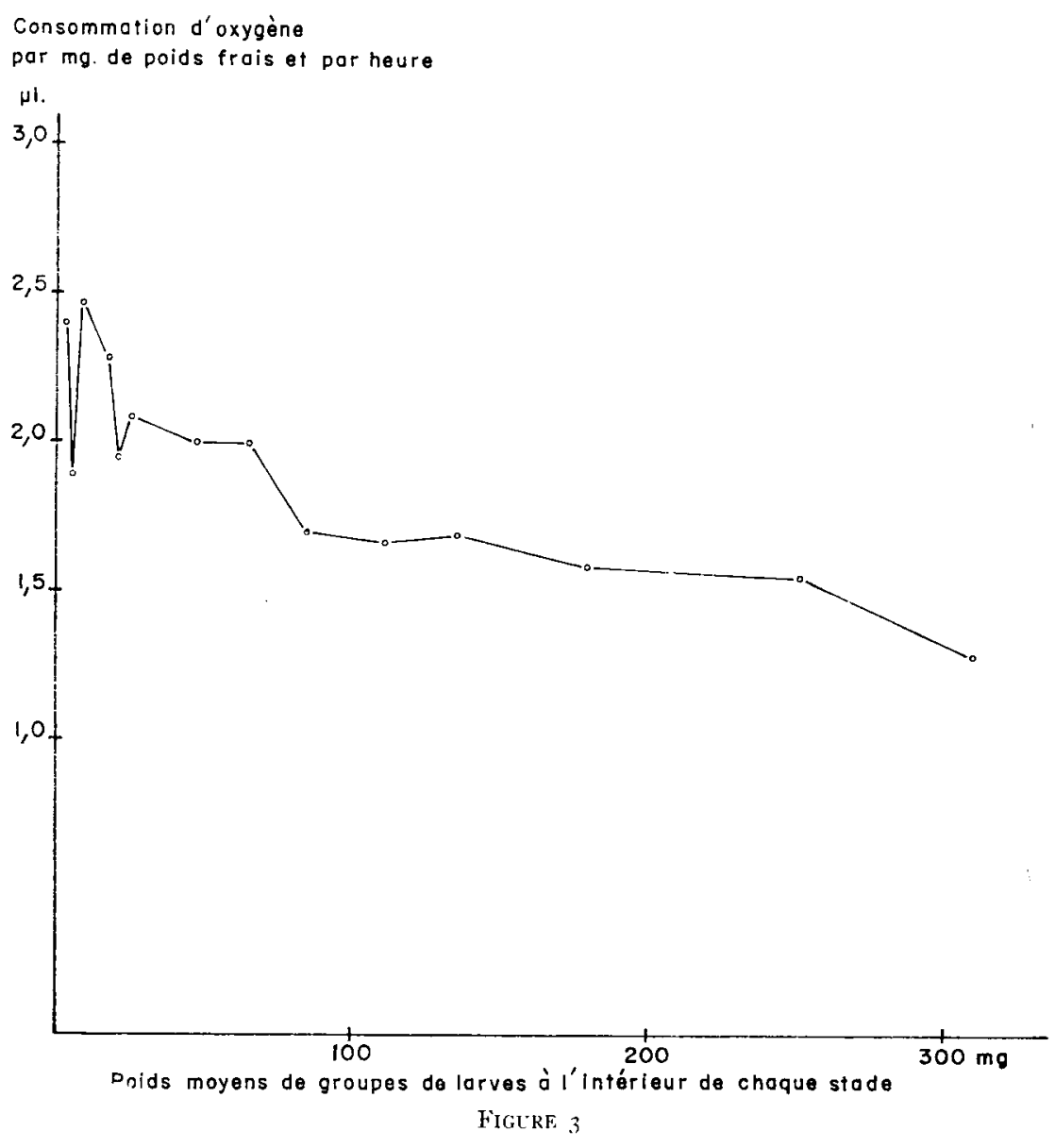

\section{DISCUSSION - CONCLUSIONS}

La méthode de Warburg nous a permis d'obtenir une évaluation périodique des échanges respiratoires des larves d'ouvrières de Bombus terrestris aux différents stades.

La détermination de la consommation moyenne d'oxygène pendant l'intermue a nécessité plusieurs expériences pour chaque stade larvaire. Il est évident que la croissance ne peut se dérouler normalement si les insectes sont maintenus dans les fioles durant plusieurs jours. En effet, chez l'espèce Bombus terrestris, chaque larve reçoit de la part de la reine et, plus ta1d, des ouvrières, une nourriture liquide régurgitée, constituée d'un mélange de pollen et de miel. Au fur et à mesure du développement, chaque larve, isolée dans son propre compartiment, est alimentée à travers un 
petit orifice pratiqué au sommet de la cellule cireuse, jusqu'à une phase proche de la nymphose. D'autre part, le séjour prolongé dans une atmosphère dépourvue de gaz carbonique peut perturber l'animal (CALVET et PRAT, I956). C'ependant, des épreuves comparatives permettent de ne pas tenir compte de ce facteur.

Certaines variations de la consommation d'oxygène, observées dans le tableau, peuvent s'expliquer par le fait que des groupes de larves ont été prélevés, pour un même stade larvaire, dans des couvées différentes, ou bien à une période proche d'une mue. En effet, des éléments nous manquent encore, permettant de caractériser exactement les stades d'intermue chez les Insectes. Bien qu'il existe une rariation relativement petite dans la durée du développement larvaire des diverses couvées, il n'est cependant pas exclu que l'on puisse observer des différences sensibles dans l'intensité respiratoire des larves en rapport avec la couvée, notamment entre les ouvrières nourrices et les butineuses.

Il convient de rappeler que chez les Bourdons, la taille des ouvrières est variable, mais ce polymorphisme est essentiellement fonctionnel. Che\% la plupart des espèces de Bourdons, les ouvrières les plus petite's prodiguent leurs soins à la progéniture, accomplissent des travaux ménagers, tandis que la récolte de provende - nectar, pollen - et la défense du nid incombent aux ouvrières de plus grande taille. La répartition des ouvrières en deux groupes, ou sous-castes - les nourrices et les butineuses - ne traduit pas toujours de façon évidente les fonctions précises des ouvrières de taille différente. Fn effet, il existe au sein d'une colonie, tous les intermédiaires entre les ouvrières les plus petites et les plus grandes, de telle sorte que les activités de ces différents individus ne peuvent être déterminées qu'en fonction de leur physiologie et de leur comportement.

Une étude plus approfondie des échanges respiratoires, en particulier des mesures de quotient respiratoire, des larves de Bombus pourrait peut-être permettre de dégager un critère mesurable dı polymorphisme des imagos. Chez A pis mollifica, Mer.ampr et WiLlis ont montré que les échanges respiratoires étaient plus importants chez les larves destinées à devenir des reines que che\% celles devant donner des ouvrières. D'après Alı.ts, les larves mâles d'Apis mellifica consomment davantage d'oxygène que les larves ouvrières, à poids égal.

Des conditions expérimentales différentes, relatives à la température, au stade d'évolution de la colonie, ne permettent pas d'établir des comparaisons bien précises avec les données obtenues chez Apis mellifica. IA plupart des auteurs n'ont considéré que les températures moyennes : $32^{\circ} \mathrm{C}$. (Al, LAMPY et WILIIS, SHUEI, et DiXON), qui coïncident à pen près avec l'optimum de développement des abeilles, mais il est délicat de comparer des résultats chez des insectes où la régulation thermique sociale joue un grand rôle (fig. 4).

Des expériences sur l'évolution des échanges respiratoires des larves d'A pis mellifica et de Bombus en fonction de la température, permettraient d'observer si l'intensité respiratoire des larves suit les oscillations de la température dans le même sens; car, chez les adultes d'Apis mellifica, il a été montré (HEUSNER et RoTh, I063) que la consommation d'oxygène augmentait quand la température diminuait. Il convient en effet de rappeler que ce phénomène est en relation avec la régulation thermique de la ruche.

Des précisions semblent également nécessaires concernant la période d'expérimentation, et par conséquent la nature de l'alimentation proposée aux larves. PAR- 


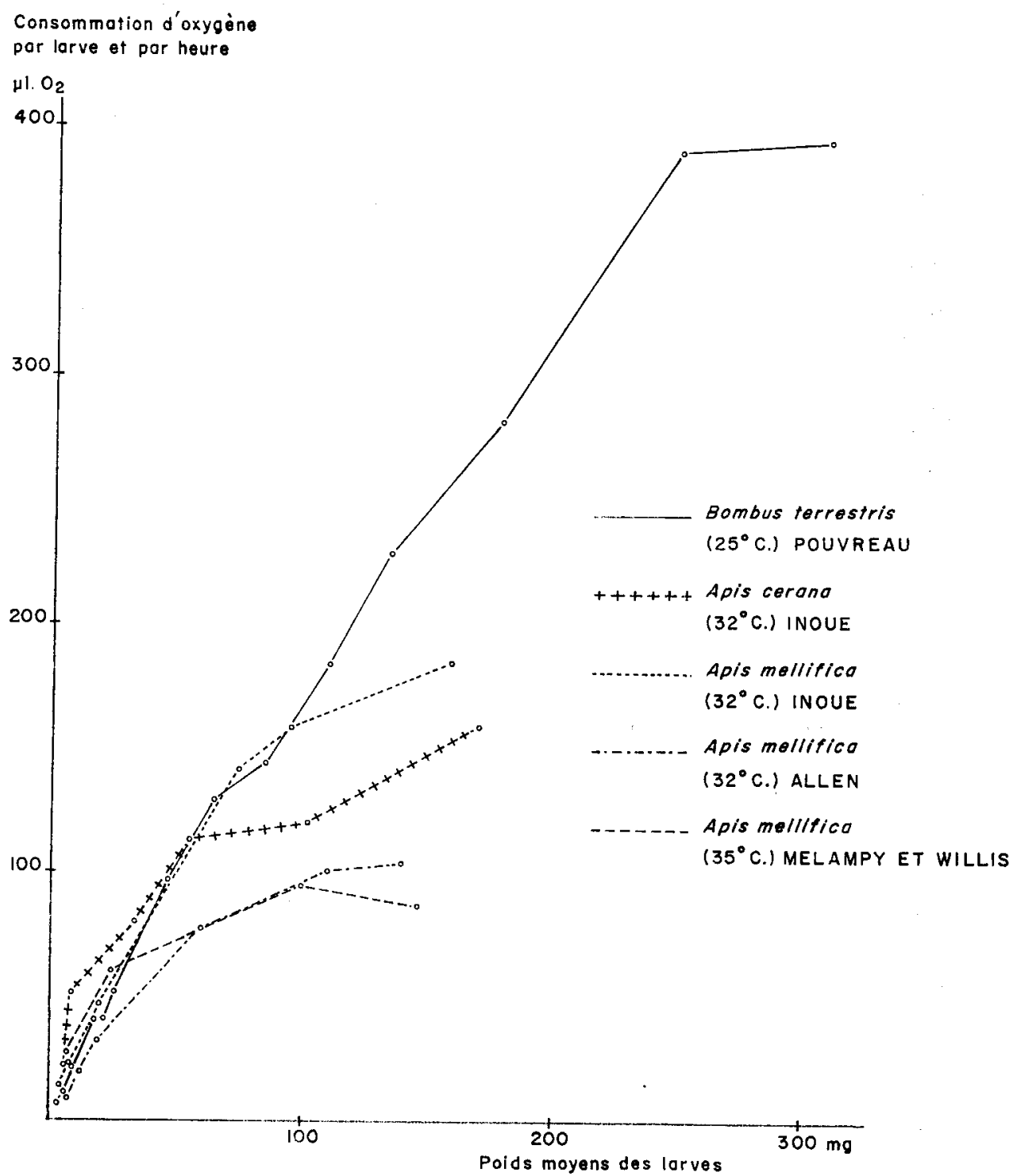

Figure 4 
HON (I 909) a pu mettre en évidence chez l'Abeille des modifications des échanges nutritifs et spécialement des échanges respiratoires pendant les différentes saisons.

\author{
Ręu pour publication $6 n$ iuillet 1968.
}

\title{
SUMMARY
}

\author{
LVOLUTION OF THE CONSIIMPTION OF OXYGEN DURING THE LARVAL DEVELOPMENT \\ OF " BOMBUS TERRESTRIS " I. WORKERS
}

The author studies the evolution of the consumption of oxygen by the worker larvate of Bombus terrestris at the different stages of development.

Experiments were carried out using Warburg's appliance and summerson constant-volume differential pressure gauges, at a temperature of $25^{\circ} \mathrm{C}$.

The results show an increase in the respiratory exchanges in relation to the increase in weight. However, as the larvaze increase in weight, so the consumption of oxygen per unit of weight of the body progressively decreases.

The curve of the larvae's consumption of oxygen versus increase in weight shows certain analogies with the curves obtained with Apis mellifica, despite different experimental conditions and a tricky interpretation of the results because of the thermal regulation pattem of these insects.

\section{REMERCIEMENTS}

J'adresse mes remerciements à M. le Professeur le Berre (liaculté des Sciences d'Orsay) pour l'aide qu'il m`a apportée dans la rédaction de ce mémoire. Je remercie également M. Arnoux, Directeur du Service de Biométrie de l'I. N. R. A., pour sa précieuse contribution à l'interprétation statistique des résultats.

\section{RÉFÉRENCES BIBLIOGRAPHIQUES}

AlLEN M. I)., 1959. Respiration rates of larvae of drone and worker honeybees, Apis mellifera L. J. econ. Enlom,l. 5.3 (3), 399-102.

Calvet F., PRAT 11, I956. Hicrocalorimétrie. Applicalions biologiqu's, $281-375$, Marson, l'aris.

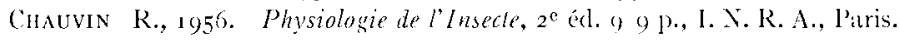

Ileusver A., Roth A., rof3. Consommation d'oxygène de l'. lbeille à différentes températures. C. K. 1cad. Sci. Paris, 256, $284-285$.

IIUKusima S., Inoue A., I $9^{6}+$. Respiration rates of japanese and european honcybees during various stages of development. Kans. - Byochn.... Kenkytuk. - Ho, 6, 36-4t.

INoue A., r964. Change of respiration rate during larval development of the japanese and european worker honeybees. Bee Science, 5 (1), 3-6.

J.EGAY J. M., al., I966. Exercices de slatislique pont biologistes, 309 p., Flammarion, Paris.

MetampY R. M., WILILS F. R., I 939. Respiratory metabolism during larval and pupal development of the female honeybee (Apis mellifica I..) Physiol. Zool., 12 (3), 302-3 I .


$9^{\mathrm{e}}$ sér. $1-5^{\mathrm{C}}$.

Renbolm H., Havser G., 1064. Sur la gelée royale des abeilles. VIII. Mise en évidence d'un principe actif, dans la nourriture larvaire des reines. Z. phy'siol. ('hemie (Hopje Seyler's), 339, $25^{1-254}$.

Siner R. W.. I)rXoN S. E., i 959. Studies in the mode of action of royal jelly in honevbee development. II. Respiration of newly emerged larvae on various substrates. Canal. J. Zool., 37, So3-81 3 .

UMbreit W. W., Burris R. II., S'TAufier J. F., 1964. Manomelric lechniques, 305 p., liurgese Publ. Comp., Minneapolis. 